\title{
Minimally Invasive Technique for Repairing CSF Leaks Due to Defects of Posterior Table of Frontal Sinus
}

\author{
Kranti Bhavana ${ }^{1} \quad$ Raj Kumar $^{1} \quad$ Amit Keshri $^{1} \quad$ Sushil Aggarwal ${ }^{1}$ \\ ${ }^{1}$ Department of Neurosurgery, Sanjay Gandhi Postgraduate Institute \\ of Medical Sciences, Lucknow, Uttar Pradesh, India \\ Address for correspondence Kranti Bhavana, MS (ENT), DNB, \\ Department of Neurosurgery, Sanjay Gandhi Postgraduate Institute of \\ Medical Sciences, Rai Bareli Road Lucknow, Lucknow, Uttar Pradesh \\ 226014, India \\ J Neurol Surg B 2014;75:183-186. \\ (e-mail: bhavana.kranti@gmail.com; kranti_bhavana@yahoo.co.in).
}

\begin{abstract}
Keywords

- CSF rhinorrhea

- frontal sinus

- posterior table fracture

- endoscopic repair

- frontal trephine

Objective Cerebrospinal fluid (CSF) leaks from the frontoethmoid and sphenoid region can be easily dealt with endoscopic approaches, but CSF rhinorrhea due to frontal sinus fractures are difficult to treat solely by the nasal endoscopic approach and may require external repair. The technique described targets defects of the posterior table of the frontal sinus where conventional osteoplastic approach of obliteration is usually done. This technique is minimally invasive and involves repair using an endoscope via a frontal trephine.

Methods We have treated five cases of traumatic CSF rhinorrhea with this technique, and the mean follow-up is 1 year (range: 10-14 months). The frontal sinus is opened by making a small stab incision (frontal trephine), and the defect site is localized by visualization via endoscope through the trephine. The repair is then performed with fat, bone graft, and fibrin glue.

Results Closure of the defect was achieved in a single stage in all the patients, and none of them had a recurrence of leak in the 1-year follow-up period.

Conclusion This is a good technique for superiorly and laterally placed posterior table defects of the frontal sinus with minimal morbidity and excellent closure rates.
\end{abstract}

\section{Introduction}

Cerebrospinal fluid (CSF) rhinorrhea is being managed by endoscopic approaches in most cases these days and hence has come under the armamentarium of otolaryngologists. Recent advances in nasal endoscopic surgery in anterior skull base area have made it the procedure of choice for the repair of CSF rhinorrhea. The results of the repair very much depend on the site of the leak, localization of the site, and materials used for repair. Trauma to the head is one of the most common reasons for CSF rhinorrhea; often the site of defect in the bone and the dura is situated in the cribriform area and the frontal sinus. CSF repair from the lateral part of the frontal sinus or from posterior table defects are challenging if endoscopic

received

July 26, 2012

accepted after revision

October 28, 2013

published online

March 3, 2014

repair is being contemplated. Intranasal access to these sites is difficult and hence leads to unsatisfactory repair and higher failure rates.

In our experience, repair of CSF rhinorrhea of frontal sinus origin can almost always be addressed endoscopically if the defect is not very large. Defects of the posterior table up to $0.5 \mathrm{~cm}$ can be repaired with the help of an endoscope with minimal morbidity. In our experience, cranialization of the frontal sinus is not required in most of the cases for management of the CSF leak due to posterior table fractures. The frontal sinus is opened by making a small stab incision just below the brow. The defect site is localized with the help of an endoscope introduced through the opening in the frontal sinus. Repair is then performed with fat, fibrin glue, bone, and

(c) 2014 Georg Thieme Verlag KG Stuttgart · New York
DOI http://dx.doi.org/ 10.1055/s-0033-1363503. ISSN 2193-6331. 


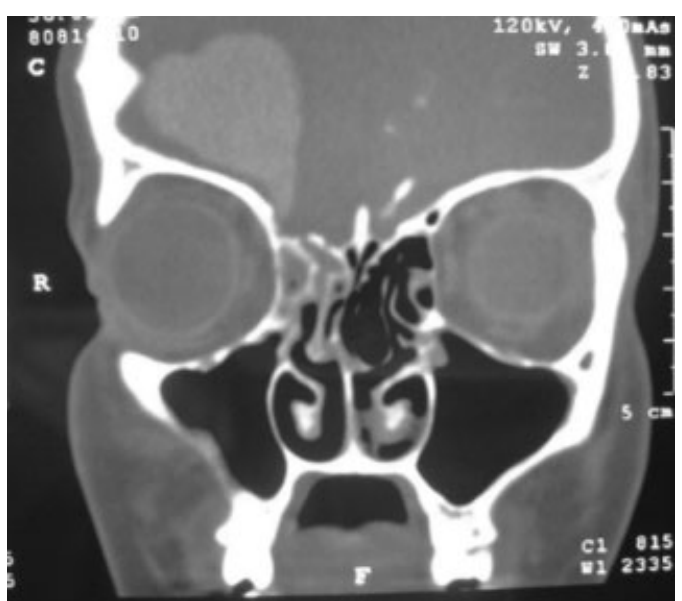

Fig. 1 Coronal computed tomography scan cut showing site of frontal sinus cerebrospinal fluid leak.

fascia. Adequate repair can most often be achieved by this minimally invasive route.

\section{Materials and Methods}

We treated five cases of traumatic CSF rhinorrhea of the frontal sinus origin in the last 3 years with a mean follow-up of 1 year (range: 10-14 months). These patients were post head injury cases with a fracture of the posterior table of the frontal sinus (-Figs. 1 and 2). Bone chips were seen in the frontal sinus in all the cases, and brain herniation was encountered in two cases. One patient had accompanying pneumocephalus as well (-Fig. 3). All these patients had posttraumatic CSF rhinorrhea where conservative treatment had failed. Our main surgical aim was to prevent further CSF leak and to maintain the frontal sinus outflow tract.

\section{Surgical Steps}

All these patients were subjected to diagnostic endoscopy before the surgical procedure. Leakage from the frontal sinus

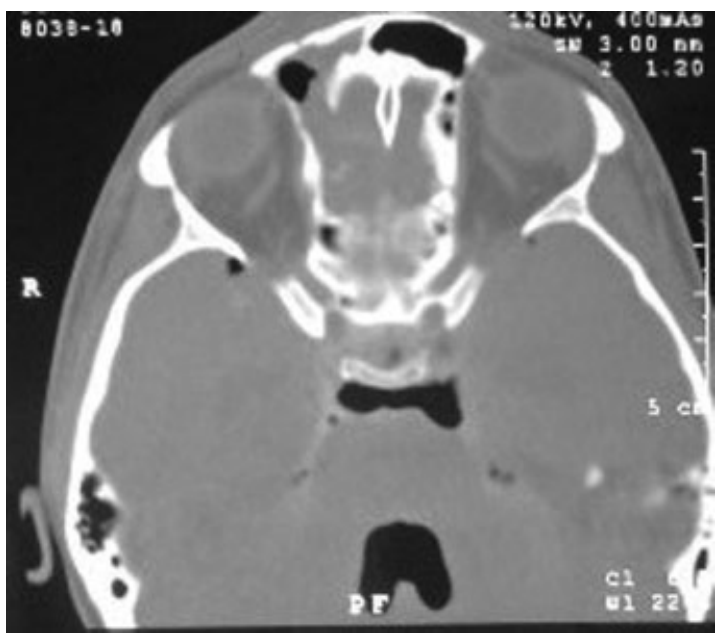

Fig. 2 Axial cut of computed tomography scan showing large defect in the posterior table of the frontal sinus.

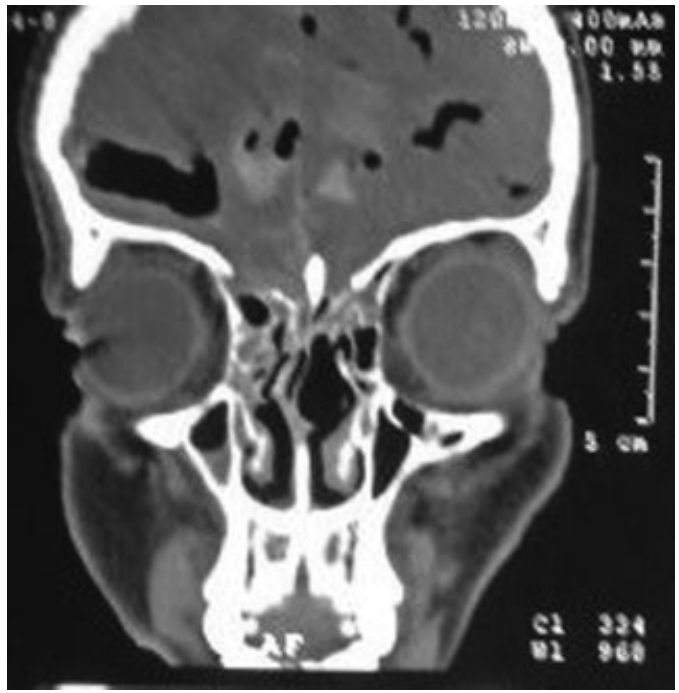

Fig. 3 Coronal computed tomography scan showing pneumocephalus due to frontal sinus cerebrospinal fluid leak.

area was confirmed. Because all these patients were post head injury cases, frank CSF leakage was seen from that area. Before proceeding to the procedure, we classified the defects of the frontal sinus radiologically as small and large defects, with a cut-off of $0.5 \mathrm{~cm}$. Defects located inferiorly in the frontal sinus and near the frontonasal duct area are usually repaired endoscopically, and those located higher up in the posterior table are dealt with using a conventional extracranial obliteration method. At our center we mainly target defects of the posterior table that cannot be adequately approached via the nasal endoscopic route and would require extracranial repair. The size of the posterior frontal table defect taken on for endoscopic repair was approximately up to $0.5 \mathrm{~cm}$.

The frontal sinus was opened by making a small stab incision halfway between the medial canthus and brow (frontal trephination) ( - Fig. 4). The opening was further enlarged with a cutting burr large enough to accommodate

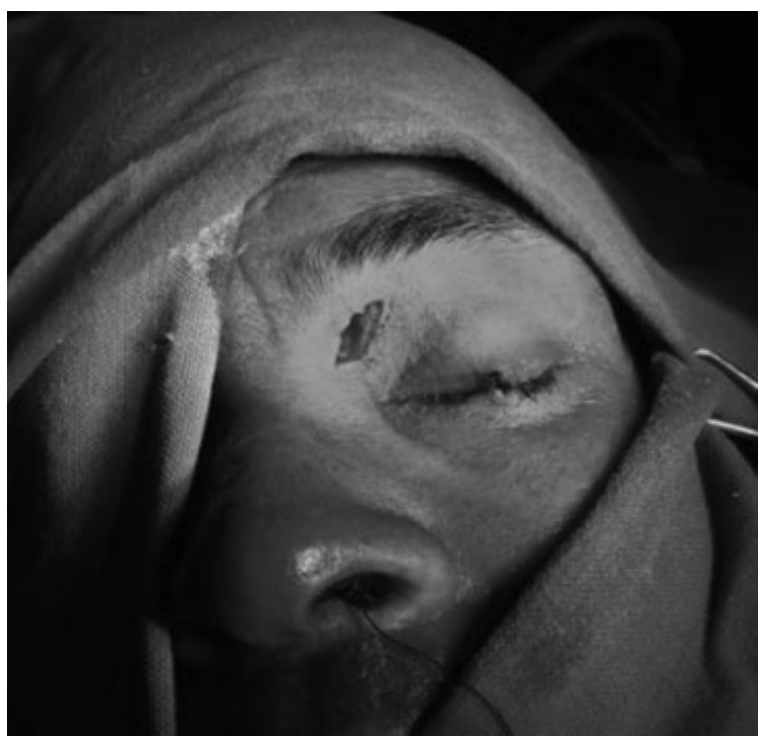

Fig. 4 Operative picture of the incision used for frontal trephination. 
a 0 degrees nasal endoscope and an endoscopic instrument. The size of this opening in the bone was $\sim 4 \mathrm{~cm}$. The frontal sinus was entered directly and the defect site visualized under greater magnification.

In all five cases we could visualize the defect and CSF was seen leaking through a bony defect in the posterior table of the frontal sinus in a pulsatile manner. Having localized the defect, we stripped the frontal sinus mucosa around the defect and removed loose bony fragments around the defect site that were often seen piercing the dura.

In three patients where the defect was $\sim 0.5 \mathrm{~cm}$ and not associated with an encephalocele, fat secured with a suture was used to plug the defect completely by pushing it through the defect and then pulling it out slightly so it would fit snugly into the defect (bath-plug technique). It was fixed in this position by layering it with fibrin glue. In two patients where the bony defect was associated with encephalocele, the edges of the defect were made raw and a piece of septal cartilage was introduced into the defect and engaged between the bony edges and the dura. Finally, the defect was layered with a fascia lata graft and layered with fibrin glue, thus ensuring a good seal of the defect. After ensuring that the leak site was well plugged above, an endoscope was introduced into the nasal cavity. Angulation of the scope was changed to 70 degrees, and the frontal sinus opening was localized. The drainage of the frontal sinus at the frontonasal duct area was evaluated and any leakage of CSF checked from there. It is important to evaluate the frontonasal duct area postoperatively so that any residual leakage site can be repaired by an overlay/onlay method. This method ensures both "above" and "below" repair of frontal sinus origin CSF leakage. In four of our patients, the leakage site was primarily from the posterior frontal table, and the frontonasal pathway was intact. One patient had leakage from the cribriform area as well that was repaired using fascia lata graft in an onlay method.

Using an endoscope for defects up to $0.5 \mathrm{~cm}$ in this manner not only gives a better visualization of the leakage site but also carries minimal morbidity because craniotomy is avoided. It is a good technique for moderate size defects of the posterior table of the frontal sinus.

\section{Results}

All five patients who had been operated on with this procedure were followed up for a median duration of 1 year. In all of them we used the technique just described to localize and seal the defect. Single-stage repair was performed in all these patients using fat, cartilage, fascia lata, and fibrin glue. In two of the patients there was accompanying encephalocele in which septal cartilage was used to seal the defect. This helped prevent further prolapse of the intracranial component into the frontal sinus. In one patient there was an associated defect of the cribriform area that was repaired using fat, fascia lata, and fibrin glue by the overlay method. The frontal sinus was not obliterated in any of these patients, and the frontal outflow tract was kept intact. We have followed up these patients for an average of 1 year, and none of them had any recurrence of CSF leak. One patient complained of neuralgic pain at the operative site that was managed conservatively by analgesics. Cosmetic considerations have been the mainstay of this approach, but one patient of the five developed a small hypertrophic scar at the incision site.

\section{Discussion}

Frontal sinus fractures comprise between 5\% and $15 \%$ of maxillofacial traumas. ${ }^{1,2}$ The incidence is $\sim 9$ per 100,000 adults per year. A third of frontal sinus fractures affect the anterior table alone; isolated fractures of the posterior table are rare. Fractures of the frontal sinus most commonly occur as the result of blunt trauma occurring during a motor vehicle accident. Gross CSF rhinorrhea occurs in $\sim 20 \%$ of patients. Approximately $60 \%$ of these patients may also present with some type of orbital trauma. ${ }^{3,4}$ The use of high-resolution 1.5$\mathrm{mm}$ thin-cut computed tomography (CT) scanning is currently the gold standard for evaluating the sinuses. CT provides essential information regarding frontal sinus anatomy, involvement of the anterior and/or posterior wall, as well as details of the degree of comminution and displacement of the fractures. It also provides visualization of any associated craniofacial and cerebral injuries. ${ }^{4,5}$ In all the cases presenting to us, CT scan was the investigation of choice. Bony defect of the posterior table was clearly seen in these cases. Brain herniation was seen in two. CT cisternography was routinely done in all our cases to see the exact site of the egress of CSF in the frontal sinus and the nasal cavity.

The main goals of treatment of frontal sinus fractures are ${ }^{6}$ the protection of intracranial structures, cessation of CSF leakage, prevention of posttraumatic infection, and restoration of facial aesthetics.

The history of frontal sinus surgery dates back to as early as 1870 when Wells operated on mucopyocele of the frontal sinus. Many surgeons have addressed the pathology of the frontal sinus using ablative or obliterative methods developed by Reidel-Schenke ${ }^{7}$ and Rohrich. ${ }^{8}$ Bergara and Itoiz devised the osteoplastic flap procedure in an attempt to improve cosmesis and surgical exposure. ${ }^{9}$ Cranialization of the frontal sinus developed by Donald and Bernstein ${ }^{10}$ has been used very frequently to treat frontal sinus fractures.

With the advent of newer instrumentation and techniques, the treatment of frontal sinus fractures and CSF leak have undergone many changes. Nasal endoscopes have added to our current armamentarium of surgical treatment. Treatment of frontal fractures associated with CSF leak differs according to the site of injury. Fractures of the posterior table of the frontal sinus that are nondisplaced and have a CSF leak are to be managed conservatively, keeping open the option of sinus exploration when the CSF leak does not subside. Current protocols state that if there is a defect more than one table width of the frontal sinus wall, the sinus has to be explored and obliteration or cranialization done accordingly.

Although the gold standard of treatment of CSF rhinorrhea by extracranial technique is obliteration, there are definite drawbacks to this procedure such as facial scarring, frontal bone embossment, frontal neuralgia, donor site morbidity, and loss of physiologic function of the frontal sinus. ${ }^{6}$ In the 
technique described here, the incision used to approach the frontal sinus is very small; the opening made in the sinus is also minimal. Hence the complications just described are not encountered.

Endoscopic repair of frontal sinus CSF leaks and encephaloceles otherwise can be an effective method if meticulous attention is directed toward preservation of the frontal sinus outflow tract, thus avoiding an osteoplastic flap and obliteration. The major limiting factor for an endoscopic approach is extreme extension superiorly or laterally within the posterior table beyond the reach of current instrumentation. ${ }^{11}$ The unilateral endoscopic approach for repair of frontal sinus CSF leak in four cases was described by Roehm and Brown in which single-stage repair could be performed in three cases. ${ }^{12}$ Endoscopic-assisted trephination approach for repair of frontal sinus cerebrospinal fluid leaks has been described where a frontal trephine was made and the endoscope was introduced through it. CSF leak of frontal sinus origin was then repaired. ${ }^{13}$

We approached the frontal sinus by creating a small window (frontal trephination), and then adequate visualization was made possible with an endoscope. The endoscopic view is magnified to improve visualization of the defect. Because the endoscope is placed directly inside the frontal sinus, posterior table defects can be identified and the dural defects better repaired. Thus this procedure is useful for managing even superiorly or laterally placed posterior frontal table defects with better results in comparison with endoscopic repair done solely via the intranasal route. After ascertaining the site of leak on the CT scan, it is easier to place the incision accordingly. Use of an endoscope and one instrument is possible via a comparatively small opening, and surgical dexterity is possible through that opening. One important aspect worth mentioning here is the need to strip the whole mucosa of the frontal sinus to avoid mucocele formation in the near future. When the defect is localized, mucosa around the defect is stripped and the repair is site specific. The fact that the site is well delineated and visualized with an endoscope makes it possible to close the defect precisely, and then there is no need to obliterate the sinus at all. Our mean follow-up range has been 1 year (range: 1014 months), and we have not encountered any cases of mucocele formation to date. We also make sure that the frontal sinus draining pathway is not compromised in any way so stenosis at that level is avoided. In one patient who had a cribriform leak in association with the posterior frontal table leak, we used fascia lata using an overlay technique. The opening of the frontal sinus was not compromised and drainage was not hampered. Because this procedure entails only a small frontal trephination, it thus has less morbidity associated compared with extra- and intracranial repair that involves osteoplastic flap elevation and obliteration. Cosmetically our procedure is well accepted by patients. In cases where there is a comminuted fracture involving the posterior frontal table and the frontal drainage area, this method may be inadequate because we would then have to block the sinus at its opening and obliterate the sinus as a whole, which is not possible via this minimally invasive route.

\section{Conclusions}

This technique is minimally invasive and offers a good approach to the difficult frontal sinus. It is advantageous in comparison with both the intracranial and nasal endoscopic method. It can deal with superiorly and laterally placed posterior frontal defects that are currently not accessible with current nasal endoscopic methods. There is an added advantage of better visualization of the bony as well as the dural defect. In comparison with the intracranial method, the complications and morbidity are much less. This method involves only a small stab skin incision, making it cosmetically more acceptable to the patient. This trephination technique is slowly being used experimentally at different centers for the repair of CSF leaks of frontal sinus origin. Their long-term results can be studied once more patients are treated by this approach and further research is performed with a larger sample size.

\section{References}

1 May M, Ogura JH, Schramm V. Nasofrontal duct in frontal sinus fractures. Arch Otolaryngol 1970;92(6):534-538

2 Gerbino G, Roccia F, Benech A, Caldarelli C. Analysis of 158 frontal sinus fractures: current surgical management and complications. J Craniomaxillofac Surg 2000;28(3):133-139

3 Gonty AA, Marciani RD, Adornato DC. Management of frontal sinus fractures: a review of 33 cases. J Oral Maxillofac Surg 1999;57(4): 372-379; discussion 380-381

4 Levine SB, Rowe LD, Keane WM, Atkins JP Jr. Evaluation and treatment of frontal sinus fractures. Otolaryngol Head Neck Surg 1986;95(1):19-22

5 Kim KS, Kim HU, Chung IH, Lee JG, Park IY, Yoon JH. Surgical anatomy of the nasofrontal duct: anatomical and computed tomographic analysis. Laryngoscope 2001;111(4 Pt 1): 603-608

6 Meyer TK, Rhee JS, Smith TL. Frontal sinus fractures. In: Kountakis SE, ed. Frontal Sinus Disease. New York, NY: Springer; 2005: 133-143

7 Reidel-Schenke H. Ueber die Stimhohlen und ihre Erkrankungen [inaugural dissertation]. Jena, Germany; 1898

8 Rohrich RJ, Hollier LH. Management of frontal sinus fractures. Changing concepts. Clin Plast Surg 1992;19(1):219-232

9 Bergara AR, Itoiz AO. Present state of the surgical treatment of chronic frontal sinusitis. AMA Arch Otolaryngol 1955;61(6): 616-628

10 Donald PJ, Bernstein L. Compound frontal sinus injuries with intracranial penetration. Laryngoscope $1978 ; 88(2$ Pt 1): 225-232

11 Strong EB, Buchalter GM, Moulthrop THM. Endoscopic repair of isolated anterior table frontal sinus fractures. Arch Facial Plast Surg 2003;5(6):514-521

12 Roehm CE, Brown SM. Unilateral endoscopic approach for repair of frontal sinus cerebrospinal fluid leak. Skull Base 2011;21(3): 139-146

13 Crozier DL, Hwang PH, Goyal P. The endoscopic-assisted trephination approach for repair of frontal sinus cerebrospinal fluid leaks. Laryngoscope 2013;123(2):321-325 